El impacto de la infraestructura en el crecimiento económico colombiano: un enfoque smithiano

Carlos Ortiz, Diana Jiménez y Gissel Cruz 
Lecturas de Economía, 90 (enero-junio 2019), pp.97-126

\author{
Carlos Ortiz, Diana Jiménezy Gissel Cruz
}

\title{
El impacto de la infraestructura en el crecimiento económico colombiano: un enfoque smithiano
}

Resumen: En este trabajo se propone una nueva forma de calcular el acervo infraestructural, el cual se compone de todos los activos durables de uso público, ya sean construidos por agentes privados o estatales. De esta forma, las regresiones del crecimiento económico para Colombia arrojan que no se puede descartar la bipótesis de rendimientos constantes a escala en el capital empresarial (agregado del capital fijo y el capital bumano) y en la infraestructura. Se estima, además, que la elasticidad producto de la infraestructura es significativamente mayor que la del capital empresarial. Una vez se controla por la acumulación de los factores productivos, los principales determinantes del crecimiento (y la productividad) son la diversificación del sector industrial manufacturero, la capacidad de compra de la población y la tasa de homicidios. El enfoque smithiano del desarrollo económico es compatible con estos halla agos. Finalmente, la descomposición del crecimiento económico nacional sugiere que este ha sido predominantemente extensivo.

Palabras clave: infraestructura, crecimiento económico, diversificación productiva, capacidad de compra, derechos de propiedad, rendimientos a escala.

Clasificación JEL: E01, E22, H54, O11, O47.

\section{The impact of infrastructure on economic growth in Colombia: a Smithian approach}

\begin{abstract}
This paper proposes a new way of calculating the infrastructural stock. This is composed of all durable assets for public use, whether built by private or state agents. In this way, economic growth regressions for Colombia yield that the bypothesis of constant returns to scale in entrepreneurial capital (aggregate of fixed and human capital) and infrastructure cannot be ruled out. It is also estimated that the product elasticity of infrastructure is significantly greater than that of entrepreneurial capital. Once controlled for the accumulation of production factors, the main determinants of growth (and productivity) are the diversification of the manufacturing industrial sector, the purchasing power of the population, and the homicide rate. The Smithian approach to economic development is compatible with these findings. Finally, the decomposition of national economic growth suggests that this has been predominantly extensive.
\end{abstract}

Keywords: infrastructure, economic growth, productive diversification, purchasing power, property rights, scale economies.

JEL Classification: E01, E22, H54, O11, O47.

\section{L'impact des infrastructures sur la croissance économique colombienne: une approche Smithienne}

Résumé: Cet article propose un nouveau mode de calcul la valeur de l'infrastructure, laquelle comprend tous les actifs durables à usage public, bien qu'ils soient construits par des agents privés ou par l'État. Les régressions de la croissance économique en Colombie montrent que l'bypothèse de rendements d'échelle constants du capital des entreprises (total du capital fixe et du capital bumain) et des infrastructures ne peut être exclue. On montre également que l'élasticité de l'infrastructure par rapport au produit est nettement supérieure à celle du capital de l'entreprise. Une fois contrôlés par l'accumulation de facteurs productifs, les principaux déterminants de la croissance (et de la productivité) sont : la diversification du secteur manufacturier industriel, le pouvoir d'achat de la population et le taux d'bomicides. L'approche théorique de Smith en matière de développement économique est compatible avec ces résultats. Enfin, on montre que la décomposition de la croissance économique nationale suggère que celle-ci a été largement extensive.

Mots-clés: infrastructure, croissance économique, diversification de la production, pouvoir d'achat, droits de propriété, rendements d'échelle.

Classification JEL: E01, E22, H54, O11, O47. 


\title{
El impacto de la infraestructura en el crecimiento económico colombiano: un enfoque smithiano
}

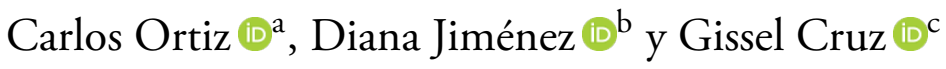

-Introducción. -I. El Enfoque smithiano del desarrollo económico. -II. Antecedentes teóricos y empíricos. -III. Una nueva estimación del acervo infraestructural de Colombia. -IV. Nuevas regresiones del crecimiento económico colombiano. -V. La productividad y la dinámica económica nacional. -Conclusiones. -Anexo estadístico. -Referencias.

doi: 10.17533/udea.le.n90a04

Primera versión recibida el 15 de mayo de 2018; versión final aceptada el 4 de agosto de 2018

\section{Introducción}

En este artículo se postula que la infraestructura de un país se compone de todos los activos durables de uso público. Por tanto, se propone una nueva forma de calcular el acervo infraestructural en Colombia. Para llevar a cabo esta tarea se desagrega la formación bruta de capital fijo en dos partes: 1) la inversión en capital fijo que sirve primordialmente a propósitos privados, y 2) la inversión en capital fijo que contribuye a generar la infraestructura nacional, esto es, el acervo de bienes duraderos, ya sean construidos por agentes

a Carlos Humberto Ortiz: profesor, Universidad del Valle. Dirección postal: Universidad del Valle, Departamento de Economía, Ciudad Universitaria Meléndez, C. 13 \#100-00, Cali (Colombia). Dirección electrónica: carlos.ortiz@correounivalle.edu.co.

https://orcid.org/0000-0003-0344-2544

b Diana Marcela Jiménez: profesora, Universidad del Valle. Dirección postal: Universidad del Valle, Departamento de Economía, Ciudad Universitaria Meléndez, C. 13 \#100-00, Cali (Colombia). Dirección electrónica: diana.marcela.jimenez@correounivalle.edu.co. https://orcid.org/0000-0002-9639-036X

c Gissel Natalia Cruæ: economista egresada, Universidad del Valle. Dirección postal: Universidad del Valle, Departamento de Economía, Ciudad Universitaria Meléndez, C. 13 \#100-00, Cali (Colombia). Dirección electrónica: gissel.cruz@correounivalle.edu.co.

https://orcid.org/0000-0002-0706-001X

Agradecemos el apoyo de la Universidad del Valle. 
Ortiz, Jiménez y Cruz: El impacto de la infraestructura en el crecimiento económico...

privados o estatales, que son de servicio público. Integrando las respectivas series de inversión con el método de Harberger (1972), se estiman los acervos de capital fijo privado y de infraestructura del país. Esta información se utiliza para estimar una nueva descomposición del crecimiento económico nacional.

En varios análisis econométricos del crecimiento económico colombiano se ha estimado que la tecnología agregada del país se caracteriza por rendimientos decrecientes a escala en el capital fijo y el capital humano (Ortiz, Uribe \& Vivas, 2013; Ortiz \& Jiménez, 2016, 2017). El enfoque metodológico utilizado en esos análisis se desarrolla en Ortiz, Uribe y Vivas (2013), y se basa en la descomposición del crecimiento económico que propone Hall (1988). Dicho enfoque se concreta en la siguiente ecuación:

$$
G P I B=\varepsilon * G+G A+u,
$$

donde $G P I B$ es la tasa de crecimiento anual del producto interno bruto, $G$ es la tasa de crecimiento ponderada de los factores de producción, $\varepsilon$ es la elasticidad de escala de los factores productivos, $G A$ es un residuo no explicado del crecimiento económico (el crecimiento de la productividad multifactorial), y $u$ es una perturbación aleatoria independientemente distribuida con media cero. Como medida de la acumulación factorial $(G)$ se utiliza el promedio ponderado de las tasas de crecimiento del capital fijo y el capital humano, donde las ponderaciones se definen, siguiendo a Hall (1988), con la participación de los factores en los costos de producción de las empresas.

Así, pues, para el caso colombiano, las regresiones de la ecuación (1) han arrojado de forma robusta y significativa que el coeficiente $\varepsilon$ es positivo pero menor que la unidad. Este resultado riñe con el principio de replicación. Si todos los factores de producción fueran replicables, la tecnología agregada de una economía en el largo plazo se debería caracterizar, al menos, por rendimientos constantes a escala $(\varepsilon=1)$ : si se duplican las empresas (y los factores), no hay razón por la cual no se duplique el producto. De hecho, los análisis del crecimiento económico de largo plazo, que suponen competencia y replicabilidad de los factores productivos, también suponen que la elasticidad de escala de la tecnología agregada es igual a 1 (e.g. Solow, 1957). Pero, si las estimaciones para Colombia arrojan que esta elasticidad es menor que 1 
de forma estadísticamente significativa, se puede sospechar que algún factor productivo no es replicable o ha sido excluido del análisis.

Ortiz y Jiménez (2017) plantean que este resultado implica que la tecnología agregada experimenta rendimientos decrecientes a escala en los factores empresariales, o bien que otros factores productivos no considerados pueden actuar como determinantes del crecimiento económico. En este sentido, un candidato obvio es el capital público representado en la infraestructura nacional.

La continuación de este artículo se estructura de la siguiente manera. En la sección I se muestra que el enfoque smithiano del desarrollo económico no solo considera el rol de los empresarios y de los trabajadores en la acumulación de capital fijo y humano, sino que también le asigna un rol fundamental al Estado como proveedor de bienes públicos, lo que incluye, por supuesto, la dotación infraestructural de un país. En la sección II se hacen referencias a la literatura internacional y nacional sobre el impacto de la infraestructura en el desarrollo económico. A continuación, en la sección IV, se explica cómo se estima el crecimiento de la infraestructura en Colombia. Posteriormente, se presentan las nuevas regresiones del crecimiento económico en la sección $\mathrm{V}$, mientras que en la sección VI se analiza el impacto del crecimiento de la productividad multifactorial en la dinámica económica nacional de largo plazo. Finalmente se presentan las conclusiones, las referencias y el anexo estadístico.

\section{El enfoque smithiano del desarrollo económico}

Según Adam Smith, la diversificación productiva (en sus palabras, la "división del trabajo") y la capacidad de compra de la población (en sus palabras, la "extensión del mercado") son los principales motores de la productividad y del crecimiento económico en el sistema capitalista. En su visión, la interacción entre la expansión de la oferta por la diversificación productiva y la expansión de la demanda por el aumento de la capacidad de compra induce el aprovechamiento de economías de escala y genera un proceso sostenido de crecimiento económico. Así, pues, para el padre de la economía política 
Ortiz, Jiménez y Cruz: El impacto de la infraestructura en el crecimiento económico...

tanto los empresarios como los consumidores -los agentes privados- juegan un rol protagónico en el desenvolvimiento económico capitalista. No obstante, nuestro autor también le asigna un papel fundamental al Estado. En este sentido, la siguiente cita de la Riqueza de las Naciones es reveladora:

Según el sistema de la libertad natural, el Soberano únicamente tiene tres deberes que cumplir, los tres muy importantes, pero claros e inteligibles al intelecto humano: el primero, defender a la sociedad contra la violencia e invasión de otras sociedades independientes; el segundo, proteger en lo posible a cada uno de los miembros de la sociedad de la violencia y de la opresión de que pudiera ser víctima por parte de otros individuos de esa misma sociedad, estableciendo una recta administración de justicia; y el tercero, la de erigir y mantener ciertas obras y establecimientos públicos cuya erección y sostenimiento no puedan interesar a un individuo o a un pequeño número de ellos, porque las utilidades no compensan los gastos que pudiera haber hecho una persona o un grupo de éstas, aun cuando sean frecuentemente muy remuneradoras para el gran cuerpo social (Smith 1776/1958, pp. 612-613).

La defensa nacional; la protección de los ciudadanos en su vida, honra y bienes; la provisión y mantenimiento de las instituciones públicas y la construcción de obras públicas son todos servicios de utilidad general que constituyen lo que hoy en día se denomina bienes públicos. Todos estos son bienes que ningún agente privado puede o quiere proveer o mantener, a pesar de su gran utilidad social, y si lo llegara a hacer no podría impedir su uso por los demás agentes ni recuperar los costos de la inversión. Smith resalta de forma meridiana que el valor social de los bienes públicos supera su valor privado, lo que justifica la intervención del Estado. Con respecto a la administración de justicia, Smith precisa que sus principales objetivos son la garantía de los derechos de propiedad y la preservación del imperio de la ley como condiciones sine qua non del desarrollo económico capitalista:

No pueden florecer largo tiempo el comercio y las manufacturas en un Estado que no disponga de una ordenada Administración de justicia; donde el pueblo no se sienta seguro en la posesión de su propiedad; 
en que no se sostenga y proteja, por obra de la ley, la buena fe de los contratos, y en que no se dé por sentado que la autoridad del Gobierno se esfuerza en promover el pago de los débitos por quienes se encuentran en condiciones de satisfacer sus deudas. En una palabra, el comercio y las manufacturas sólo pueden florecer en un Estado en que exista cierto grado de confianza en la justicia del Gobierno (Smith 1776/1958, p. 808).

Estas citas muestran que, en la visión smithiana, la provisión y el mantenimiento de bienes públicos de todo orden son funciones esenciales del Estado, y especial énfasis se pone en el respeto a los derechos de propiedad como fundamento institucional de la economía capitalista.

\section{Antecedentes teóricos y empíricos}

El trabajo de Aschauer (1989) mostró de forma pionera que la inversión pública en la infraestructura era un factor determinante de la productividad en los Estados Unidos. Utilizando su enfoque metodológico, Cárdenas, Escobar y Gutiérrez (1995) encontraron que la infraestructura tiene impactos positivos y significativos en la productividad y el crecimiento económico de Colombia, y concluyeron que los rezagos del desarrollo infraestructural del país son el principal obstáculo para el desarrollo económico.

Barro (1990) recogió el enfoque de Aschauer y resolvió un simple pero revelador modelo de equilibrio económico general donde el impacto externo del gasto público sobre la productividad del capital empresarial es fundamental para generar crecimiento sostenido. Este autor propone la siguiente tecnología Cobb-Douglas en su modelo: $y=A g^{\alpha} k^{1-\alpha}$, donde $y$ es el producto del período de análisis, A es la productividad multifactorial, k es el capital empresarial (síntesis de todas las formas de capital que manejan las empresas), $g$ es el gasto público del período, $1-\alpha$ es la elasticidad producto del capital, y $\alpha$ es la elasticidad producto del gasto público. Se supone que $\alpha$ es una fracción positiva y que, por tanto, la economía experimenta rendimientos constantes a escala en el capital y el gasto público. Nótese que para las empresas el gasto 
Ortiz, Jiménez y Cruz: El impacto de la infraestructura en el crecimiento económico...

público actúa como una externalidad positiva. Si se supone, como lo hace Barro, que ese gasto público se financia contemporáneamente con un impuesto sobre la renta, entonces tenemos que $g=\tau y$, donde $\tau$ es la tasa impositiva, la tecnología se hace lineal en el capital empresarial: $y=(\tau A)^{1 /(1-\alpha)} k$, lo que hace constante la productividad marginal del capital, y permite así lograr una tasa de crecimiento económico constante (Rebelo, 1991).

Así, pues, las externalidades productivas del gasto público pueden jugar un rol fundamental en la explicación de la dinámica económica de los países. Aplicando ese enfoque de externalidades para Colombia, Sánchez, Rodríguez y Núñez (1996) estimaron que la elasticidad-producto del capital público en la producción manufacturera es mayor que la elasticidad-producto del mismo capital en la producción agrícola, siendo ambas estimaciones positivas y significativas; estas elasticidades miden el impacto externo de la acumulación de capital público sobre la productividad de ambos sectores.

Por su parte, Easterly y Rebelo (1993) encontraron que la inversión en transporte y comunicaciones está estrechamente vinculada al crecimiento económico, pero los efectos particulares de la tributación en cada país son variables.

En esta línea de argumentación, Posada y Gómez (2002) mostraron que la tasa de crecimiento económico colombiana se relaciona positivamente con el gasto público en capital humano e infraestructura física. El trabajo de estos autores se centró en estimar el nivel óptimo del gasto público, como se plantea en Barro (1990), y sugirieron que las tasas impositivas en Colombia inducen distorsiones en la asignación de los recursos que financian el gasto público en Colombia.

No obstante, utilizando un análisis de vectores autoregresivos, Perdomo (2002) encontró que la inversión pública en electricidad, gas, agua, educación, minería e industria manufacturera tiene un impacto positivo y significativo en el crecimiento económico colombiano.

Más recientemente, Palei (2015) analiza los hallazgos internacionales sobre la productividad y la competitividad de los países, y concluye que ambas están determinadas por las instituciones, la infraestructura, el ambiente ma- 
croeconómico, la salud, la educación primaria, la preparación tecnológica, el tamaño del mercado, el desarrollo del mercado financiero y otros factores. Todos estos son bienes públicos o bienes meritorios (bienes privados con impactos externos) que el Estado debe proveer, erigir o mantener; en particular, el desarrollo infraestructural se mide por esta autora con la calidad de las carreteras, la infraestructura ferroviaria, la infraestructura del transporte aéreo y la oferta de energía eléctrica.

Así, pues, existe un acuerdo general entre los analistas económicos sobre el impacto decisivo de la infraestructura nacional sobre la productividad y el crecimiento económico de los países.

\section{Una nueva estimación del acervo infraestructural de Colombia}

El aporte metodológico de este trabajo radica principalmente en la desagregación de la formación bruta de capital fijo (FBKF) que reportan las diferentes cuentas nacionales de Colombia en el período 1950-2014. La formación bruta de capital fijo privado (FBKFP) comprende la inversión en los siguientes rubros: vivienda, mejora de tierras y desarrollo de plantaciones y huertas, equipo de transporte, y maquinaria y equipo. Nótese que, a diferencia de la infraestructura, estos bienes son generalmente demandados y utilizados por las empresas y los hogares -los agentes privados- como bienes de uso exclusivo. Por otra parte, la inversión que contribuye a la acumulación del acervo infraestructural se denomina formación bruta de capital fijo infraestructural (FBKFI). Sean estatales o privados, estos bienes cumplen la función de prestar servicios públicos. La Tabla 1 muestra los componentes de la FBKF que se clasifican como inversión infraestructural según la fuente de las cuentas nacionales y el año base.

Estos bienes representan el conjunto de activos estatales y privados que prestan servicios como infraestructura, o sea, como bienes duraderos de uso público. La mayoría de ellos son bienes estatales (la sola inversión en obras civiles representó en 2014 el 70,6 \% del gasto en infraestructura, tal como se define arriba). Ello no impide que algunas construcciones no residenciales sean privadas y que también se puedan considerar parte de la dotación infraestructural del país. Un centro comercial, un centro de convenciones, una 
Ortiz, Jiménez y Cruz: El impacto de la infraestructura en el crecimiento económico...

torre de parqueaderos, una universidad, un colegio, una biblioteca, un estadio, etc., pueden ser activos privados, pero ofrecen servicios públicos que potencian el desarrollo económico y social. Sin embargo, algunas construcciones no residenciales pueden ser de uso exclusivamente privado como, por ejemplo, una bodega, una fábrica, un silo, etc., y, en este sentido, no hacen parte de la infraestructura nacional. No es posible diferenciar en las cuentas nacionales el gasto en construcciones no residenciales que son de uso exclusivamente privado del gasto en aquellas que son de uso público, pero es lícito suponer que al gasto en construcciones de uso privado representa una fracción menor de la inversión que aquí se denomina infraestructural (FBKFI). El acervo correspondiente de capital infraestructural (KI) que se estima a continuación incluirá, por tanto, una pequeña fracción de activos privados, pero su tasa de crecimiento estará fuertemente correlacionada con el crecimiento del verdadero acervo infraestructural, y eso es lo que se requiere para las regresiones de crecimiento económico.

Tabla 1. Componentes de la formación bruta de capital fijo infraestructural en Colombia

\begin{tabular}{l|l}
\hline Cuentas Nacionales & Rubros por código \\
\hline $\begin{array}{l}\text { Banco de la República (1958), } \\
\text { según López, Gómez y Rodrí- } \\
\text { guez (1996) }\end{array}$ & $\begin{array}{l}\text { - Otras edificaciones. } \\
\text { - Otras construcciones. }\end{array}$ \\
\hline DANE (1975) & $\begin{array}{l}\text { 2. Otros edificios (excluye vivienda). } \\
\text { 3. Otras construcciones salvo las que tienen por finalidad } \\
\text { mejorar tierras. }\end{array}$ \\
\hline \multirow{2}{*}{ DANE (1994) } & $\begin{array}{l}\text { 39.03. Edificios no residenciales. } \\
\text { 40. Trabajos y obras de ingeniería civil. }\end{array}$ \\
\hline DANE (2005) & $\begin{array}{l}\text { 410200. Trabajos de construcción y construcción de } \\
\text { edificaciones no residenciales. }\end{array}$ \\
& $\begin{array}{l}\text { 410300. Trabajos de reparación, adecuación y manteni- } \\
\text { miento de edificaciones. }\end{array}$ \\
& 4201. Obras civiles. \\
\hline
\end{tabular}

Fuente: procesamiento propio. 
Una vez se define para cada año la FBKFI se halla la FBKFP por residuo $(F B K F P=$ FBKF - FBKFI), y se procede a definir la distribución de la inversión entre estos dos rubros. Así, la distribución entre FBKFI y FBKFP de 1950 a 1964 se calcula según las cuentas nacionales con base en 1958; la de 1965 a 1993, según las cuentas nacionales con base en 1975; la de 1994 a 2004, según las cuentas nacionales con base en 1994 y, finalmente, la del período 2005-2014 se calcula según las cuentas nacionales con base en 2005.

Las series del producto interno bruto (PIB) y de la FBKF a precios de 2005 se proyectan para el período 1950-2014 como se explica a continuación. Utilizando las series retropoladas del DANE con base en 2005 para el período 1975-2014, se proyectan las series del PIB y de la FBKF empalmándolas hasta 1950 con las mismas variables que reportan las cuentas nacionales del DANE con base en 1975 (1965-1975), y también las cuentas nacionales del Banco de la República con base en 1958 (1950-1964). Con la composición de la inversión entre infraestructura y bienes privados se definen las series de inversión en FBKFI y en FBKFP a precios de 2005.

Dado que ya se cuenta con las series de inversión arriba mencionadas, se calculan a continuación los correspondientes acervos de capital: el KFP y el acervo de KI. Para ello, se estima inicialmente el siguiente indicador:

$$
\kappa=i /(\delta+g),
$$

donde $\kappa$ es la razón esperada capital-producto, $i$ es la razón esperada del esfuerzo de inversión (participación de la inversión en el producto), $\delta$ es la tasa de depreciación del capital, y $g$ es la tasa de crecimiento promedio anual del producto en el período de análisis. El método se le debe a Harberger (1972). La Tabla 2 muestra los parámetros requeridos para calcular el coeficiente $\kappa$ de cada forma de capital.

Ya que durante el período 1950-1985 se tuvo una mayor estabilidad del crecimiento económico (la volatilidad aumenta posteriormente), se calcularon los parámetros $g$ e $i$ con las series del PIB, la FBKFI y la FBKFP del período mencionado. El parámetro $g$ es común a los dos cálculos y se estima como la tasa de crecimiento promedio anual del PIB en el período 1950-1985. El coeficiente inversión-producto, $i$, se estima para cada año del periodo analizado para las dos series de inversión-producto (FBKFI/PIB y FBKFE/PIB); 
Ortiz, Jiménez y Cruz: El impacto de la infraestructura en el crecimiento económico...

en la Tabla 2 se reporta el promedio anual del período considerado para las dos series; las desviaciones estándar de estas dos series son pequeñas: 0,017 y 0,012 , respectivamente. Por otra parte, la tasa de depreciación, $\delta$, se calcula para cada año del período 1965-1995 con las cuentas nacionales con base en 1975.

En la Tabla 2 se reporta el promedio de la tasa de depreciación del período analizado para cada tipo de acervo (KI y KFP). Para hacerlo se definen, en primer lugar, los componentes del capital; en segundo lugar, se determinan las tasas de depreciación de cada rubro del capital como el inverso de la vida útil estimada (depreciación lineal); finalmente, se calcula el promedio ponderado de la tasa de depreciación, donde las ponderaciones se definen por la contribución de cada rubro de la inversión a su correspondiente agregado, según las cuentas nacionales con base en 1975.

Tabla 2. Parámetros de las series de inversión y del producto

\begin{tabular}{lcccc}
\hline Serie & $g$ & $i$ & $\delta$ & $\kappa$ \\
\hline FBKFP & 0,04683595 & 0,14193548 & 0,10306058 & 0,94688965 \\
FBKFI & 0,04683595 & 0,071946947 & 0,15154439 & 0,36267176 \\
\hline
\end{tabular}

Fuente: procesamiento de los autores con base en las cuentas nacionales.

Así, pues, para definir la tasa de depreciación de la infraestructura se supone que la vida útil de los edificios no residenciales es de 30 años (la tasa de depreciación esperada es $1 / 30$ ), y la vida útil de las demás construcciones civiles es de 6 años (la tasa de depreciación esperada es 1/6). Para definir esta última tasa se tuvo en cuenta el gran peso de las carreteras en la inversión y el hecho de que los inviernos, la baja calidad de las obras y los atentados sistemáticos de las guerrillas y otros actores armados irregulares disminuyen la durabilidad de las carreteras y de toda la infraestructura nacional. ${ }^{1}$ A continuación se calcula la tasa de depreciación de la infraestructura para cada año del período 1965-1995; con el promedio del período se estima la tasa de depreciación del capital infraestructural que se reporta en la Tabla

1 Poveda (2005) menciona que las lluvias tropicales en la geografía montañosa de Colombia "disuelven" las carreteras aproximadamente cada cinco años. 
1: $15,15 \%$. Esta tasa es cercana a la tasa de depreciación de las carreteras y otras obras civiles que estiman Pachón y Ramírez (2006): 13\%. Se probaron diferentes supuestos sobre la vida útil de estas construcciones (entre 5 y 9 años) y las estimaciones finales de las regresiones de crecimiento no variaron significativamente.

Por otra parte, para definir la tasa de depreciación del capital fijo privado se supone una vida útil de 30 años para las viviendas, de 20 años para la mejora de tierras, de 10 años para el equipo de transporte y de 7 años para la maquinaria y el equipo. Teniendo en cuenta la participación de estos rubros en la FIBKFP, se estima una tasa de depreciación del capital privado para cada año del período 1965-1995, cuyo promedio es de 10,3\%, como se muestra en la Tabla 1. Con estos parámetros se estima la razón esperada capital-producto $(\kappa)$ para ambas formas de capital (KI y KFP).

Con el PIB de 1950 se estiman los acervos de infraestructura y del capital fijo privado del mismo año. Posteriormente, con la fórmula del inventario perpetuo y las correspondientes series de inversión, se calcula la serie completa de los acervos de capital mencionados. ${ }^{2}$ Dadas las series de KI y KFP, se calculan las tasas de crecimiento de los correspondientes acervos de capital: GI y GKFP. Finalmente, se estima el crecimiento promedio de los factores que son demandados por las empresas (capital fijo privado y capital humano) siguiendo la metodología de Hall (1988):

$$
G K E=s * G K F P+(1-s) * G K H,
$$

donde GKE es la tasa de crecimiento del capital empresarial, GKFP es la tasa de crecimiento del capital fijo privado, $G K H$ es la tasa de crecimiento del capital humano, y $s$ es la participación del capital fijo privado en los costos de producción de las empresas. ${ }^{3}$

2 La fórmula del inventario perpetuo dice que $K_{t}=(1-\delta) K_{t-1}+I_{t-1}$, donde $K_{t}$ es el acervo de capital en el período $t, I_{t-1}$ es la inversión del período anterior, y $\delta$ es la tasa de depreciación.

3 La tasa de crecimiento del capital humano $(G K H)$ se muestra en los anexos de Ortiz y Jiménez (2017). Ese capital se estima con la metodología de Hall y Jones (1999), y consiste básicamente en el empleo multiplicado por un factor que crece exponencialmente con los a nos de escolaridad de la fuerza laboral. 
Ortiz, Jiménez y Cruz: El impacto de la infraestructura en el crecimiento económico...

El Gráfico 1 muestra las sendas temporales del capital fijo privado (KFP: línea continua medida a la izquierda) y del capital infraestructural (KI: línea doble medida a la derecha) entre 1950 y 2014. Se observa que entre 1950 y 1978 ambos acervos evolucionaron pari passu, pero luego se abrió una brecha que apenas se volvió a cerrar hacia 2010.

Gráfico 1. Capital fijo privado e infraestructura en Colombia, 1950-2014

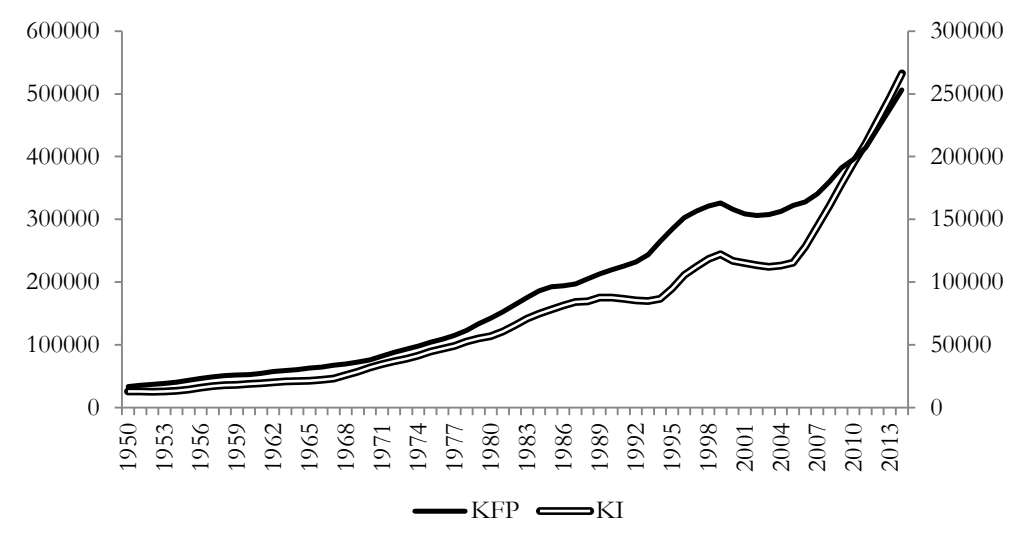

Fuente: procesamiento de los autores con base en las cuentas nacionales.

Dicho lo anterior, conviene relativizar, pues parecería que al cerrarse la brecha contable entre ambas formas de capital ya se soluciona el déficit, pero no es así. Esta información solo cuenta lo que se ha hecho, pero no puede contar lo que no. Es decir, no revela, por ejemplo, que Colombia desmontó de forma progresiva su sistema ferroviario desde los años 50, que también abandonó los cables aéreos para el transporte de carga, que, con la notable excepción de Medellín, no se construyeron los metros que requerían las grandes ciudades, que tampoco se construyeron los tranvías urbanos y los trenes de cercanía que requerían las regiones, que el sistema de carreteras, después de casi 40 años, apenas ahora se va a actualizar, que las vías terciarias son deficientes, que las fuerzas armadas irregulares han atentado sistemáticamente contra la infraestructura nacional (carreteras, puentes, oleoductos, torres de energía) y que la ineficiencia estatal y la corrupción han aplazado y encarecido las obras de infraestructura, y eso cuando los recursos no han sido desviados hacia otros propósitos. 


\section{Nuevas regresiones del crecimiento económico colombiano}

El impacto de la diversificación productiva y de la capacidad de compra de la población en el crecimiento económico nacional se exploró en un par de artículos previos (Ortiz y Jiménez, 2016, 2017). Los resultados estadísticos no permitieron rechazar la hipótesis de que, una vez se controla por la acumulación de capital fijo y capital humano, las variables mencionadas tienen impactos positivos y significativos sobre el crecimiento económico. En esos análisis, la infraestructura pública quedó subsumida en el capital fijo, de manera que no se midió su impacto específico sobre el crecimiento económico nacional. Esta omisión se debió al carácter multidimensional de la infraestructura, los cambios de la calidad de los diferentes tipos de infraestructura, la carencia de información confiable y a que los errores de medición hacen difícil construir un indicador adecuado de la acumulación del capital infraestructural. Sin embargo, la omisión en una regresión de una variable explicatoria que debe ser incluida puede generar sesgos en la estimación de los parámetros. Por lo tanto, uno de los objetivos de este trabajo es examinar cómo cambian los resultados obtenidos en las pasadas investigaciones cuando se incluye una estimación del crecimiento de la infraestructura nacional en las regresiones de crecimiento. También se inquiere sobre los propios impactos del crecimiento de la infraestructura nacional en el crecimiento económico nacional.

Las regresiones del crecimiento económico nacional utilizan como variable dependiente la tasa de crecimiento anual del PIB (GPIB). Se incluye una constante de regresión $(\mathrm{C})$, y se utiliza una variable ficticia para los períodos recesivos (R), la cual toma el valor de 1 en 1999 y 2009, y de 0 en cualquier otro año del período 1951-2014. ${ }^{4}$ La variable GKE es la tasa de crecimiento ponderada de los factores fijos empresariales (capital fijo privado y capital humano) y la variable GI es la tasa de crecimiento del acervo de infraestructura. Como índice de la variación anual de la capacidad de compra real de la población urbana se utiliza el número de metros cuadrados licenciados anualmente para construcción de vivienda por ciudadano en las primeras cinco ciudades

4 El año 2009 no fue estrictamente un año recesivo en la economía colombiana, pero la tasa de crecimiento sí disminuyó considerablemente por efecto de la recesión financiera internacional. 
Ortiz, Jiménez y Cruz: El impacto de la infraestructura en el crecimiento económico...

del país: Bogotá, Medellín, Cali, Bucaramanga y Barranquilla [M2PC5]. ${ }^{5}$ Como un indicador indirecto del respeto a la vida (la propiedad fundamental) y de los demás derechos de propiedad, se utiliza la tasa de homicidios por 100.000 habitantes (HOM).

Finalmente, se utiliza la distribución del PIB entre los siguientes sectores de la actividad económica: sector primario: minería (MIN), y actividades agropecuarias, pesca y silvicultura (VIVOS). Sector manufacturero: agroindustria (AGROIND), materias primas manufacturadas (MATPRIM), y bienes de capital (BK). Sector terciario: construcción (CONST), servicios de transporte (TRANSP), servicios públicos (PUB), comercio (CIO), servicios del gobierno (GOB), servicios financieros y afines (FIN), y servicios de alquiler y otros servicios empresariales y personales (ALQUILER).

Ya que la suma de estas variables en cada año da 100\%, se excluye la variable ALQUILER en las regresiones para evitar problemas de multicolinealidad. Como se explicó en Ortiz, Uribe y Vivas (2013), el objeto de incluir la distribución de la estructura económica nacional es capturar posibles efectos externos sobre el crecimiento económico asociados a la transformación estructural del país. En estas regresiones se examina tanto el efecto directo de la acumulación de la infraestructura nacional en el crecimiento económico como los efectos indirectos de la misma acumulación. Como se explicó arriba, el efecto directo se estima con la variable GI, mientras que los efectos indirectos se estiman con la interacción multiplicativa de esta variable con la composición sectorial del PIB. Todas estas variables se reportan en los dos anexos de Ortiz y Jiménez (2017), y las variables GPIB, GKFP, GKH, GKE y GI se presentan en el anexo estadístico de este trabajo.

Se verificó que todas las variables utilizadas en las regresiones fueran estacionarias. Sin embargo, no pasaban la prueba de estacionariedad algunas participaciones de los sectores económicos en el PIB, pues estas variables experimentan saltos bruscos debido a los cambios metodológicos de las cuentas nacionales. No obstante, es evidente que las participaciones de los sectores en el producto no pueden ser variables que deriven libremente -su suma no puede superar el $100 \%-$.

5 Ver Ortiz y Jiménez (2017) para una sustentación microeconómica de la variable M2PC5 como una medida de la variación de la capacidad de compra de la población urbana. 
El problema de la estimación de series de tiempo con órdenes de integración diferentes se resuelve corriendo las regresiones mediante el método de mínimos cuadrados ordinarios completamente modificados (FMOLS). Se estima previamente que la inclusión de tres rezagos de la variable dependiente es suficiente para corregir el problema de correlación entre las perturbaciones con el método mencionado. Tal como se muestra en la Tabla 3, se corren dos regresiones del crecimiento económico. En ambos casos, se estima que la perturbación del crecimiento parece seguir un proceso autoregresivo de tercer orden: la estimación del coeficiente asociado a GPIB(-3) parece ser negativo y significativo en todas las regresiones. Las pruebas estadísticas no rechazan la hipótesis de que los residuales de las regresiones estimadas son estacionarios. Estos resultados sustentan la validez de las inferencias estadísticas que se comentan a continuación. ${ }^{6}$

Tabla 3. Regresiones del crecimiento económico colombiano. Variable dependiente: GPIB

\begin{tabular}{|c|c|c|}
\hline Variable & Regresión 1 & Regresión 2 \\
\hline $\mathrm{R}$ & $\begin{array}{l}-3,961302^{* * *} \\
(0,769886)\end{array}$ & $\begin{array}{l}-3,868098^{* * *} \\
(0,622244)\end{array}$ \\
\hline GKE & $\begin{array}{l}0,353096^{* * *} \\
(0,096553)\end{array}$ & $\begin{array}{l}0,3293892^{* * *} \\
(0,079924)\end{array}$ \\
\hline GI & $\begin{array}{l}4,350840 \\
(2,641064)\end{array}$ & $\begin{array}{l}0,585468^{* * *} \\
(0,160401)\end{array}$ \\
\hline $\mathrm{M}_{2} \mathrm{PC} 5^{2}$ & $\begin{array}{l}6,767788^{* *} \\
(2,138021)\end{array}$ & $\begin{array}{l}7,030845^{* * *} \\
(1,035429)\end{array}$ \\
\hline VIVOS & $\begin{array}{l}-0,263930 \\
(0,282493)\end{array}$ & $\begin{array}{l}-0,315995^{* * *} \\
(0,065979)\end{array}$ \\
\hline VIVOS*GI & $\begin{array}{l}-0,039003 \\
(0,046417)\end{array}$ & \\
\hline MIN & $\begin{array}{l}-0,553775 \\
(0,975340)\end{array}$ & \\
\hline $\mathrm{MIN}^{*} \mathrm{GI}$ & $\begin{array}{l}0,103026 \\
(0,134555)\end{array}$ & \\
\hline AGROIND & $\begin{array}{l}0,228211 \\
(0,459659)\end{array}$ & \\
\hline AGROIND*GI & $\begin{array}{l}0,078596 \\
(0,082318)\end{array}$ & $\begin{array}{l}0,065097^{* * *} \\
(0,013556)\end{array}$ \\
\hline MATPRIM & $\begin{array}{l}3,353079^{* * *} \\
(1,216635)\end{array}$ & $\begin{array}{l}1,028622^{* * *} \\
(0,196982)\end{array}$ \\
\hline MATPRIM*GI & $\begin{array}{l}-0,488739^{* *} \\
(0,180622)\end{array}$ & \\
\hline
\end{tabular}

6 La información completa está disponible en este trabajo y en el anterior para quien quiera replicar los resultados. 
Ortiz, Jiménez y Cruz: El impacto de la infraestructura en el crecimiento económico...

Tabla 3. Continuación

\begin{tabular}{|c|c|c|}
\hline Variable & Regresión 1 & Regresión 2 \\
\hline BK & $\begin{array}{l}0,907196 \\
(2,008497)\end{array}$ & \\
\hline $\mathrm{BK}^{*} \mathrm{GI}$ & $\begin{array}{l}0,001543 \\
(0,223707)\end{array}$ & \\
\hline CONST & $\begin{array}{l}0,014888 \\
(0,685841)\end{array}$ & \\
\hline $\mathrm{CONST}^{*} \mathrm{GI}$ & $\begin{array}{l}-0,005762 \\
(0,089321)\end{array}$ & \\
\hline TRANSP & $\begin{array}{l}-2,266329 \\
(1,605191)\end{array}$ & \\
\hline TRANSP*GI & $\begin{array}{l}0,253863 \\
(0,202739)\end{array}$ & $\begin{array}{l}-0,194469^{* * *} \\
(0,026629)\end{array}$ \\
\hline PUB & $\begin{array}{l}-1,947351 \\
(1,255714)\end{array}$ & $\begin{array}{l}-1,167699^{* * *} \\
(0,261437)\end{array}$ \\
\hline PUB*GI & $\begin{array}{l}0,118189 \\
(0,177122)\end{array}$ & \\
\hline $\mathrm{CIO}$ & $\begin{array}{l}1,032730^{* *} \\
(0,427362)\end{array}$ & $\begin{array}{l}0,648919^{* * *} \\
(0,121835)\end{array}$ \\
\hline $\mathrm{CIO}^{*} \mathrm{GI}$ & $\begin{array}{l}-0,175108^{* *} \\
(0,068946)\end{array}$ & \\
\hline GOB & $\begin{array}{l}0,742926 \\
(0,686035)\end{array}$ & \\
\hline FIN & $\begin{array}{l}-0,524932 \\
(0,892920)\end{array}$ & \\
\hline GOB*GI & $\begin{array}{l}-0,122174 \\
(0,084585)\end{array}$ & \\
\hline $\mathrm{FIN}^{*} \mathrm{GI}$ & $\begin{array}{l}-0,050961 \\
(0,099693)\end{array}$ & \\
\hline $\mathrm{HOM}$ & $\begin{array}{l}-0,035205^{*} \\
(0,019492)\end{array}$ & $\begin{array}{l}-0,045704^{* * *} \\
(0,010048)\end{array}$ \\
\hline GPIB(-1) & $\begin{array}{l}0,042340 \\
(0,079161)\end{array}$ & \\
\hline GPIB(-2) & $\begin{array}{l}0,078468 \\
(0,070644)\end{array}$ & \\
\hline GPIB(-3) & $\begin{array}{l}-0,191075^{* * *} \\
(0,067428)\end{array}$ & $\begin{array}{l}-0,216657^{* * *} \\
(0,059641)\end{array}$ \\
\hline $\mathrm{C}$ & $\begin{array}{l}-11,07181 \\
(15,04990)\end{array}$ & $\begin{array}{l}-0,641197 \\
(2,736473)\end{array}$ \\
\hline Período & 1951-2014 & $1951-2014$ \\
\hline $\mathrm{R}^{2}$ ajustado & 0,612135 & 0,721879 \\
\hline
\end{tabular}

Nota: significación al $10 \%\left({ }^{*}\right)$, al $5 \%\left(^{(*)}\right.$ y al $1 \%\left(^{(* *}\right)$.

Fuente: procesamiento de los autores.

La primera regresión se corre con todas las variables disponibles. Posteriormente, se eliminan de forma secuencial los regresores que no son significativos al $1 \%$; de esta manera se llega a la regresión 2 . Como es de esperar, la variable ficticia para las recesiones $(\mathrm{R})$ parece tener un impacto negativo 
y significativo en el crecimiento económico. Por otro lado, las tasas de acumulación de GKE y GI muestran un impacto positivo y significativo en el crecimiento económico nacional (los coeficientes estimados, respectivamente, son: 0,329 y 0,586$)$.

En consecuencia, dado que la suma de los coeficientes asociados a la expansión del capital empresarial y a la expansión de la infraestructura arroja un valor cercano a $1(0,915)$, y que este estimativo no es estadísticamente diferente a la unidad, no se puede rechazar la hipótesis de que la economía colombiana experimenta rendimientos constantes a escala en el capital empresarial (capital fijo privado y el capital humano) y en la dotación infraestructural. Así se resuelve la aparente paradoja que motivó este artículo y se deduce, por tanto, que la omisión del crecimiento de la infraestructura en los anteriores ejercicios sesga hacia arriba la estimación de la elasticidad producto del capital empresarial.

Por otra parte, dado que la elasticidad producto de la infraestructura es significativamente mayor que la elasticidad producto del capital empresarial $(0,585>0,329)$, se deduce que el rezago nacional en la provisión de bienes infraestructurales ha podido incidir en el bajo nivel de crecimiento nacional y en la desaceleración económica de largo plazo que vive el país desde principios de la década de los 70 (la tendencia del crecimiento económico de Colombia se muestra posteriormente).

Continuando con el análisis de la regresión 2, también se encuentra que la expansión de la capacidad de compra de la población urbana (medida por los metros cuadrados construidos al año y por habitante en las cinco ciudades más grandes del país: M2PC5) parece tener un impacto positivo y significativo en el crecimiento económico nacional; nótese además que esta variable es más significativa como regresor cuando se eleva al cuadrado, lo que sugiere que la expansión de la capacidad de compra de la población acelera el crecimiento económico nacional. Este resultado es consistente con la visión smithiana sobre el rol dinámico de la demanda efectiva.

El sector de las actividades primarias dedicadas a la explotación de seres vivos (VIVOS: sector agropecuario, silvicultura y pesca) da muestras de tener un impacto negativo y significativo en el crecimiento económico de largo 
Ortiz, Jiménez y Cruz: El impacto de la infraestructura en el crecimiento económico...

plazo; este resultado sugiere que la productividad en estas actividades primarias ha estado relativamente estancada.

El impacto multiplicativo de la fracción que aporta la agroindustria al PIB con el crecimiento de la infraestructura (AGROIND*GI) parece tener un impacto positivo y significativo en el crecimiento económico nacional; esto da indicios de que la construcción de vías y la provisión de energía y otros servicios públicos potencian la expansión de la agroindustria e impactan positivamente en el crecimiento económico.

Así mismo, la contribución del sector productor de materias primas industriales (MATPRIM) al PIB también muestra un impacto positivo y significativo sobre el crecimiento económico nacional, lo que es consistente con la visión smithiana que resalta el impacto dinámico de la diversificación productiva manufacturera sobre la productividad multifactorial. ${ }^{7}$

La contribución de las actividades de transporte al producto en interacción con el crecimiento de la infraestructura (TRANSP*GI) parece tener un impacto negativo y significativo sobre el crecimiento económico nacional. Este resultado captura muy posiblemente los efectos negativos del gran rezago del sistema nacional de transporte sobre el crecimiento económico nacional, el alto costo del transporte (los peajes colombianos son exorbitantes en la región) y los efectos negativos de la ineficiencia y la corrupción en la dotación infraestructural del sistema de transporte nacional.

El impacto de la contribución de los servicios públicos (PUB) al producto también da muestras de tener un efecto negativo y significativo en el crecimiento económico, lo cual puede sugerir que la ineficiencia y la carestía de los servicios públicos afectan negativamente el crecimiento económico nacional. De otra parte, la actividad comercial (CIO) se relaciona positivamente con el crecimiento económico.

\footnotetext{
7 Se ha sugerido que más que la diversificación de las actividades manufactureras per se es la diversificación de las actividades intensivas en tecnología e inteligencia (capital humano) las que comandan la expansión de la productividad multifactorial y el crecimiento económico (Haussman, Hwang \& Rodrik, 2006; Rodrik, 2007).
} 
Finalmente, como es de esperar, la tasa de homicidios por 100.000 habitantes $(\mathrm{HOM})$ parece tener un impacto negativo y significativo sobre el crecimiento económico nacional; esto indica que la paz es rentable.

\section{La productividad y la dinámica económica nacional}

Todos estos esfuerzos analíticos se dirigen en últimas a entender la dinámica económica nacional, y por ello es necesario conocer cómo se ha comportado. Con las series del PIB de la CEPAL (1957), del Banco de la República (1958), y del DANE (1950 hasta la actualidad) se construye la serie de 1925 a 2017 a precios de 2005, y también, por supuesto, se deriva la serie de la tasa de crecimiento nacional: GPIB (ver el anexo estadístico). Esta última serie es volátil y está sujeta a ciclos, así que en el Gráfico 2 solo se presentan la tendencia de la tasa de crecimiento estimada con el filtro de Hodrick y Prescott y la tasa de crecimiento promedio anual del PIB del período 1925-2017.

Gráfico 2. Tendencia del crecimiento económico colombiano, 1925-2017

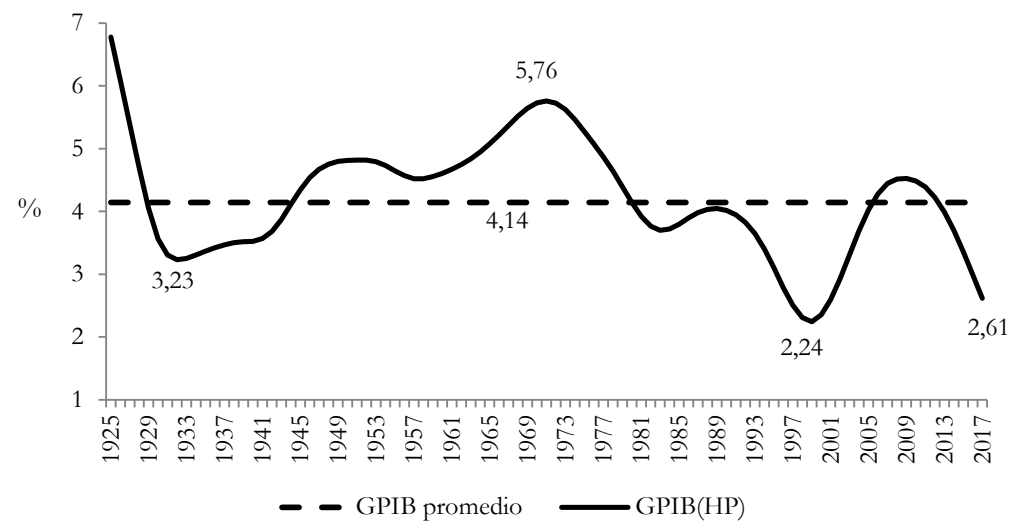

Fuente: procesamiento de los autores con base en CEPAL (1957), Banco de la República (1958) y DANE.

En el casi último siglo del desarrollo económico nacional (1925-2017) la tasa de crecimiento promedio anual ha sido del $4,14 \%$, un crecimiento mediocre en el contexto mundial; un crecimiento así no estaría mal si la dinámica 
Ortiz, Jiménez y Cruz: El impacto de la infraestructura en el crecimiento económico...

económica tendiera a aumentar, pero en la actualidad ocurre todo lo contrario. Como muestra el Gráfico 2, después de la gran recesión internacional de los años 30, la economía colombiana experimenta una leve tendencia al crecimiento acelerado: entre 1932 y 1971 la tasa de crecimiento de largo plazo pasa de 3,23\% a 5,76\%. A partir de 1971 hay un quiebre estructural en la dinámica económica nacional: se abandona la política de apoyo a la diversificación industrial y, a cambio, se promueven los sectores "líderes" de la construcción y de las exportaciones. Sin embargo, como muestran las regresiones del crecimiento económico nacional, ni la construcción ni las exportaciones (concentradas en bienes agrícolas, mineros y agroindustriales) lideran el crecimiento de largo plazo; en cambio, la diversificación productiva del sector industrial manufacturero productor de materias primas sí se correlaciona de forma positiva y significativa con el crecimiento económico nacional. Por tanto, a partir de 1971 se frena la diversificación productiva manufacturera -el sector industrial manufacturero pierde participación en el producto nacionaly se disminuye el motor que jalonó la dinámica económica nacional en las primeras siete décadas del siglo XX (Ortiz, 2016).

Además, otros motores complementarios del crecimiento económico, como el aprendizaje en la práctica en sectores intensivos en tecnología, y las externalidades asociadas al conocimiento científico y tecnológico, también son disminuidos. A partir de los años 90 el país se abre unilateralmente al comercio internacional. Dadas sus ventajas comparativas, el país se especializa todavía más en actividades intensivas en recursos naturales y fuerza de trabajo poco calificada, y se profundiza la desindustrialización nacional. En consecuencia, en las últimas tres décadas del siglo XX el país experimenta una tendencia pronunciada a la desaceleración económica. Es así como para 1999 la tasa de crecimiento de largo plazo cae al menor nivel en todo el período analizado: $2,24 \%$. La tendencia a la desaceleración económica no es tanto el resultado de la apertura per se, que era inevitable, sino del predominio del modelo neoliberal y el rechazo dogmático a la política industrialista. ${ }^{8}$ La recuperación de la dinámica económica en el siglo XXI se relaciona estrechamente con el auge

8 Sin desconocer que la influencia de los industriales llevó al abuso del proteccionismo y de la caza de rentas estatales, con consecuencias significativas en el retraso tecnológico del país (Roberts, 1989), los economistas ortodoxos se fueron al otro extremo y expresaban gustosos que "la mejor política industrial es la que no existe". 
económico mundial, el boom de los precios del petróleo y de otros minerales, y la contención de la guerrilla. Pero la recesión financiera internacional de 20082009 y la destorcida hacia la baja de los precios mencionados que comienza en 2014 detienen finalmente el auge económico y reaparece la tendencia a la desaceleración.

Con la información procesada en la regresión 2 es posible estimar el crecimiento de la productividad multifactorial (el coeficiente GA de la ecuación 1) como sigue:

$$
\widehat{G A}=G P I B-(0,3294 * G K E+0,5855 * G I) .
$$

La información estimada permite calcular que la tasa de crecimiento promedio de la productividad multifactorial en el período 1951-2014 fue casi nula $(0,1 \%)$, mientras que la tasa de crecimiento promedio anual fue del 4,31\%. Estos resultados sugieren que el crecimiento económico nacional ha sido primordialmente extensivo: se explica casi totalmente por la expansión de los factores productivos (capital privado fijo, capital humano e infraestructura). La tendencia de la variable GA se estima por medio del filtro de Hodrick y Prescott $[\mathrm{GA}(\mathrm{HP})]$ y se presenta en el Gráfico 3 junto con la tendencia del crecimiento económico nacional [GPIB $(\mathrm{HP})]$.

El Gráfico 3 permite deducir que la tendencia a la desaceleración económica nacional (línea gruesa continua) se relaciona estrechamente con la pérdida tendencial de la dinámica asociada a la innovación tecnológica (línea de triángulos): las pocas pero consistentes ganancias en productividad multifactorial que se lograron en las décadas de los años 50 y 60 (asociadas a la política de diversificación industrial), se disminuyen en las últimas tres décadas del siglo XX, y se tornan negativas en el siglo XXI con la profundización de la dependencia nacional a las actividades intensivas en recursos naturales: los sectores minero y agroindustrial (las principales exportaciones del país pertenecen a estos sectores, especialmente al sector minero; y en la parte agroindustrial no se pueden dejar de lado las exportaciones de cocaína, marihuana y heroína). Los ajustes lineales de las tendencias analizadas se pueden interpretar como las tendencias de muy largo plazo, y se presentan para resaltar la estrecha asociación entre la desaceleración económica y la disminución del crecimiento de la productividad. 
Ortiz, Jiménez y Cruz: El impacto de la infraestructura en el crecimiento económico...

Gráfico 3. Tendencias del crecimiento económico y del crecimiento de la productividad multifactorial colombiana, 1951-2014

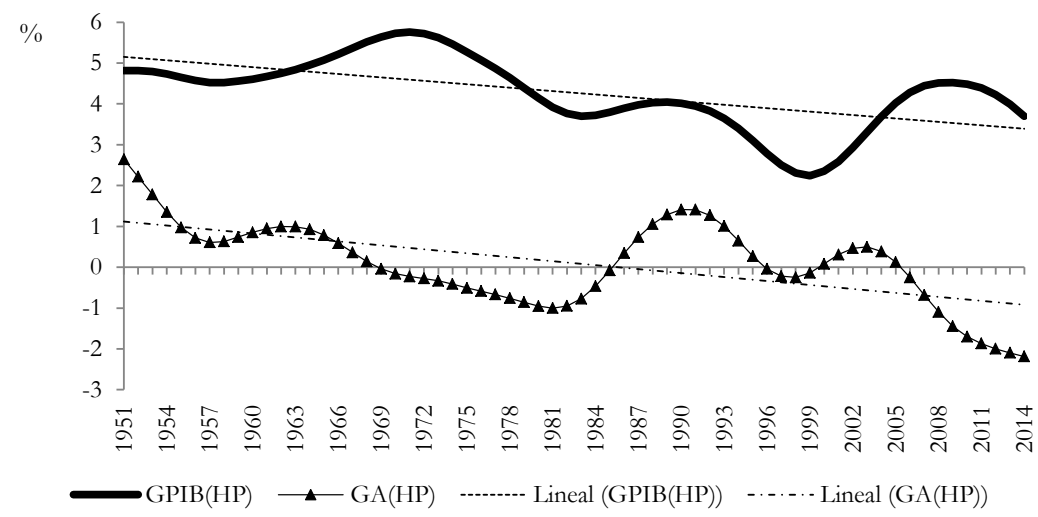

Nota: la tendencia del crecimiento del PIB se calcula con la información disponible entre 1925 y 2017.

Fuente: procesamiento de los autores.

\section{Conclusiones}

Este trabajo se motivó por la insatisfacción de los autores con un resultado contraintuitivo: la aparente existencia de rendimientos decrecientes a escala en la tecnología agregada de la economía colombiana. Se propone entonces una distribución alternativa de la inversión nacional (la formación bruta de capital fijo) en inversión fija privada e inversión en infraestructura. Se calculan a continuación los correspondientes acervos de capital con el método de Harberger. El capital que comandan las empresas se calcula como un promedio ponderado del capital fijo privado y el capital humano, y se encuentra que las regresiones del crecimiento económico nacional ahora sí son compatibles con la existencia de una tecnología agregada con rendimientos constantes a escala en el capital empresarial y la infraestructura. Se revela, además, que el impacto de la infraestructura en el crecimiento económico nacional de largo plazo es significativamente mayor que el impacto del capital empresarial, siendo ambos positivos; en consecuencia, se deduce que el rezago en la provisión nacional de infraestructura ha disminuido el crecimiento económico nacional. 
Finalmente, se encuentra que el crecimiento económico nacional en el largo plazo ha sido predominantemente extensivo: el aporte del crecimiento de la productividad multifactorial no solo ha sido escaso (el país no ha asimilado la importancia del desarrollo educativo, cultural, científico y tecnológico), sino que, además, viene disminuyendo, y ello condiciona la tendencia de largo plazo a la desaceleración económica del país.

Es hora de cambiar el modelo de desarrollo económico. El análisis de los determinantes del crecimiento económico nacional permite postular que la diversificación productiva nacional -preferiblemente intensiva en inteligencia y tecnología- y el aumento de la capacidad de compra de la población son condiciones sine qua non de una transformación productiva dinámica. También es fundamental que el Estado invierta eficientemente, de forma directa o con intermediación privada, en la infraestructura del país. Para toda esta transformación productiva se debe contar con un contexto institucional de paz y respeto a los derechos de propiedad. Finalmente, el Estado debe liderar un gran acuerdo nacional para lograr las transformaciones requeridas.

\section{Anexo estadístico}

Tabla A1. Colombia, 1950-2014

\begin{tabular}{cccccccccccc}
\hline AÑO & PIB & GPIB & FBKFI & KI & GKI & FBKFP & KFP & GKFP & GKH & $s$ & GKE \\
\hline 1950 & 35155.69 & & 1907.04 & 12749.98 & & 5513.98 & 33288.56 & & & & \\
1951 & 36252.56 & 3.1200 & 1880.65 & 12724.83 & -0.1972 & 5091.41 & 35371.80 & 6.2581 & 3.9136 & 0.4454 & 4.9578 \\
1952 & 38540.08 & 6.3099 & 2006.22 & 12677.11 & -0.3751 & 5391.07 & 36817.77 & 4.0879 & 5.0346 & 0.4402 & 4.6179 \\
1953 & 40883.33 & 6.0800 & 2418.40 & 12762.18 & 0.6711 & 5858.70 & 38414.38 & 4.3365 & 2.2904 & 0.4361 & 3.1827 \\
1954 & 43712.45 & 6.9200 & 3118.78 & 13246.54 & 3.7953 & 7200.54 & 40314.07 & 4.9453 & 3.5628 & 0.4315 & 4.1593 \\
1955 & 45421.60 & 3.9100 & 3593.07 & 14357.88 & 8.3896 & 7355.70 & 43359.82 & 7.5550 & 3.7059 & 0.4400 & 5.3994 \\
1956 & 47265.73 & 4.0600 & 3643.46 & 15775.10 & 9.8706 & 7527.13 & 46246.84 & 6.6583 & 3.2855 & 0.4461 & 4.7900 \\
1957 & 48319.76 & 2.2300 & 3398.98 & 17027.93 & 7.9418 & 7194.67 & 49007.74 & 5.9699 & 1.8309 & 0.4550 & 3.7140 \\
1958 & 49508.41 & 2.4600 & 3006.27 & 17846.42 & 4.8068 & 6011.20 & 51151.65 & 4.3746 & 1.4282 & 0.4595 & 2.7822 \\
1959 & 53087.88 & 7.2300 & 3406.98 & 18148.16 & 1.6908 & 6026.93 & 51891.13 & 1.4457 & 5.1557 & 0.4458 & 3.5018 \\
1960 & 55354.73 & 4.2700 & 3377.12 & 18804.89 & 3.6187 & 7529.00 & 52570.14 & 1.3085 & 1.5121 & 0.4387 & 1.4228 \\
1961 & 58172.30 & 5.0900 & 3706.38 & 19332.23 & 2.8043 & 8390.68 & 54681.23 & 4.0158 & 4.9204 & 0.4362 & 4.5258 \\
1962 & 61319.39 & 5.4100 & 3829.29 & 20108.92 & 4.0176 & 7265.06 & 57436.42 & 5.0387 & 4.7314 & 0.4353 & 4.8651 \\
1963 & 63336.80 & 3.2900 & 3353.12 & 20890.81 & 3.8883 & 7537.80 & 58782.05 & 2.3428 & 2.1718 & 0.4330 & 2.2458 \\
\hline & & & & & & & & & & & Continúa
\end{tabular}


Ortiz, Jiménez y Cruz: El impacto de la infraestructura en el crecimiento económico...

Tabla A1. Continuación

\begin{tabular}{|c|c|c|c|c|c|c|c|c|c|c|c|}
\hline AÑO & IB & IB & FI & KI & KI & $P$ & KFP & $P$ & $\mathrm{H}$ & $s$ & $\mathrm{KKE}$ \\
\hline 64 & 7244.69 & 1700 & 3578.41 & 21078.05 & 8963 & 740.46 & 60261.73 & 2.5172 & 5.3052 & 4245 & 1218 \\
\hline 65 & 69665.49 & 3.6000 & 3764.45 & 21462.20 & 1.8225 & 8363.00 & 62791.59 & 4.1981 & 4.4082 & 0.4259 & 4.3188 \\
\hline 1966 & 73313.69 & 5.2367 & 4515.77 & 21974.18 & 2.3855 & 9290.51 & 64683.25 & 3.0126 & 5.6803 & .4207 & 4.5581 \\
\hline 67 & 6345.14 & 1349 & 6110.63 & 23159.88 & 5.3959 & 908.55 & 67307.46 & .0570 & .5103 & .4205 & .1606 \\
\hline 1968 & 80874.77 & 5.9331 & 6852 & 25760.76 & 1.2301 & 10236.16 & 69279.26 & 2.9295 & 5.6375 & 4135 & 5178 \\
\hline 1969 & 85810.17 & 6.1025 & 7553.34 & 28709.74 & 11.4476 & 11153.42 & 72375.46 & 4.4692 & 4.6665 & .4097 & 4.5856 \\
\hline 1970 & 91137.09 & 2078 & 7455.98 & 1912.28 & 1.1549 & 13514.97 & 76069.82 & 1044 & 359 & 15 & 641 \\
\hline 1971 & 96569.53 & 5.9607 & 7550.19 & 34532.14 & 8.2095 & 14439.73 & 81744.99 & 7.4605 & & 108 & 1883 \\
\hline 1972 & 103975.59 & 7.6691 & 7528.90 & 36849.17 & 98 & 14027.81 & 87760.04 & 83 & 29 & 0.4128 & 5.5582 \\
\hline 1973 & 110965.81 & 6.7229 & 8432.77 & 38793.79 & 5.2772 & 14998.68 & 92743.24 & 5.6782 & 2.1924 & 4195 & 3.6548 \\
\hline 1974 & 117341.65 & 57458 & 9454.88 & 41347.58 & 5830 & 16010.94 & 8183.75 & 5.8662 & 3.2203 & 39 & 3419 \\
\hline 17) & 20067.77 & 2.0202 & 8998.42 & 44536.47 & . & 15491.80 & 04075.82 & 6.001 & & 4 & 6.0101 \\
\hline 1976 & 125643.36 & 4.6437 & 9402.55 & 46785.64 & 5.0502 & 17367.06 & 108841.51 & 4.5791 & 3.6727 & .4328 & 4.0650 \\
\hline 1977 & 30909.19 & 4.1911 & 1064.70 & 49098.09 & 4.9427 & 20053.44 & 14991.30 & & & & 6.4099 \\
\hline 1978 & 142045.80 & 8.5071 & 10401.51 & 52722.25 & 7.3815 & 22868.31 & 123193.67 & 7.1330 & 7.3397 & .4166 & 7.2536 \\
\hline 1979 & 149760.27 & 5.4310 & 10141.03 & 55134.00 & 4.5744 & 23023.48 & 133365.57 & 8.2568 & 3.2290 & .4156 & 5.3186 \\
\hline o & 5984.49 & & & & & 24473.80 & & & & & 2558 \\
\hline 1981 & 159838.39 & 2.4707 & 14253.77 & 60591.38 & 6.4505 & 27384.02 & 152417.11 & 6.8512 & 4.8266 & & 5.6674 \\
\hline 1982 & 161640.25 & 1.1273 & 15275.58 & 65662.87 & 8.3700 & 28582.90 & 164092.93 & 7.6604 & -1.3381 & .4194 & 2.4359 \\
\hline 1983 & 164406.07 & 1.7111 & 14759.91 & 7098 & & 28155.43 & 1757 & & & & 516 \\
\hline 1984 & 169362.80 & & 14615.37 & 74989.75 & & 25771.55 & 185805.38 & & & & 3.5886 \\
\hline 1985 & 74480.80 & 3.0219 & 14903.30 & 78240.84 & & 21589.04 & & & & & 3.8954 \\
\hline 1986 & 1845 & 5.7693 & & & & & & & & & 3.3517 \\
\hline 1987 & 94889.54 & 5.6042 & 3249.29 & 84147.41 & 3.5187 & 28340.11 & 197073.87 & 1.4877 & 8.2786 & 0.4855 & 4.9815 \\
\hline 1988 & 203689.36 & 4.5153 & 15782.17 & 84644.64 & 0.5909 & 29138.28 & 205103.43 & & & 0.4847 & 4.4681 \\
\hline 1989 & 210921.94 & 3.5508 & & & & & & 3.9006 & 03 & & 4.6772 \\
\hline 1990 & 20167.63 & 4.3835 & 12126.00 & 87686.35 & 0.0993 & 29015.04 & 219408.14 & & & 0.4511 & 2.3061 \\
\hline 1991 & 226308.59 & 2.7892 & 11863.02 & 86523.97 & -1.3256 & 29670.02 & 225810.85 & & & & 4.8635 \\
\hline 1992 & 236093.30 & 4.3236 & 12555.43 & 85274.77 & -1.4438 & 35206.35 & 232208.67 & 2.8333 & 1.7447 & 0.3901 & 2.1694 \\
\hline 1993 & 249613.87 & 5.7268 & 14541.03 & 84907.29 & -0.4309 & 46337.76 & 243483.45 & 4.8555 & & & 4.6252 \\
\hline 1994 & 263611.74 & 5.6078 & 21349.80 & 86581.09 & 1.9713 & 47207.20 & 264727.67 & 8.7251 & 1.6743 & 0.5213 & 5.3501 \\
\hline 1995 & 277650.23 & 5.3254 & 25262.06 & 94810.02 & 9.5043 & 47374.83 & 284651.88 & & 1.6722 & & 4.6393 \\
\hline 1996 & 282059.46 & 1.5881 & 22503.06 & 105704.15 & 11.4905 & 41521.22 & 302690.31 & 6.3370 & -0.7732 & 0.4553 & 2.4641 \\
\hline 1997 & 291069.90 & 3.1945 & 23140.56 & 12188.34 & 6.1343 & 40521.21 & 313016.09 & 3.4113 & 3.6071 & 0.3962 & 3.5296 \\
\hline 1998 & 292828.71 & 0.6043 & 21844.55 & 118327.39 & 5.4721 & 37928.70 & 321277.68 & 2.6393 & 2.5075 & 0.5236 & 2.5766 \\
\hline 1999 & 277627.99 & -5.1910 & 13281.44 & 122240.09 & 3.3067 & 23407.50 & 326095.31 & 1.4995 & 0.0821 & 0.3129 & 0.5257 \\
\hline 2000 & 284760.81 & 2.5692 & 15773.26 & 116996.73 & -4.2894 & 25513.71 & 315895.24 & -3.1279 & 7.6769 & 0.2712 & 4.7468 \\
\hline
\end{tabular}

Continúa 
Tabla A1. Continuación

\begin{tabular}{|c|c|c|c|c|c|c|c|c|c|c|c|}
\hline AÑO & PIB & GPIB & FBKFI & KI & GKI & FBKFP & KFP & GKFP & GKH & $s$ & GKE \\
\hline 2001 & 289538.89 & 1.6779 & 15886.62 & 115039.79 & -1.6726 & 28878.82 & 308852.61 & -2.2294 & 2.1976 & 0.3244 & 0.7614 \\
\hline 2002 & 296788.55 & 2.5039 & 15957.67 & 113492.77 & -1.3448 & 33315.34 & 305900.90 & -0.9557 & 1.7408 & 0.2843 & 0.9742 \\
\hline 2003 & 308418.23 & 3.9185 & 18017.85 & 112251.25 & -1.0939 & 36850.50 & 307689.91 & 0.5848 & 6.1776 & 0.1944 & 5.0905 \\
\hline 2004 & 324865.99 & 5.3329 & 19325.19 & 113258.05 & 0.8969 & 41679.31 & 312829.71 & 1.6704 & 1.6624 & 0.1923 & 1.6640 \\
\hline 2005 & 340156.00 & 4.7066 & 30328.93 & 115419.62 & 1.9085 & 38454.07 & 322268.61 & 3.0173 & 3.8493 & 0.2278 & 3.6597 \\
\hline 2006 & 362938.00 & 6.6975 & 35246.37 & 128257.35 & 11.1227 & 46734.63 & 327509.49 & 1.6262 & 0.6013 & 0.1651 & 0.7705 \\
\hline 2007 & 387983.00 & 6.9006 & 38509.11 & 144067.04 & 12.3265 & 54146.89 & 340490.80 & 3.9636 & 0.2104 & 0.2151 & 1.0178 \\
\hline 2008 & 401744.00 & 3.5468 & 41681.87 & 160743.59 & 11.5756 & 59470.13 & 359546.52 & 5.5965 & 2.8697 & 0.1238 & 3.2073 \\
\hline 2009 & 408379.00 & 1.6515 & 43935.85 & 178065.67 & 10.7762 & 53050.15 & 381961.58 & 6.2343 & 4.8393 & 0.2041 & 5.1240 \\
\hline 2010 & 424599.00 & 3.9718 & 45062.89 & 195016.67 & 9.5195 & 59098.11 & 395646.54 & 3.5828 & 5.4517 & 0.1662 & 5.1410 \\
\hline 2011 & 452578.00 & 6.5895 & 50489.57 & 210525.88 & 7.9528 & 73326.43 & 413969.09 & 4.6310 & 5.1996 & 0.2429 & 5.0615 \\
\hline 2012 & 470880.00 & 4.0439 & 52478.23 & 229111.43 & 8.8282 & 76687.77 & 444631.62 & 7.4070 & 3.9247 & 0.3053 & 4.9878 \\
\hline 2013 & 494124.00 & 4.9363 & 56734.18 & 246869.11 & 7.7507 & 79723.82 & 475495.40 & 6.9414 & 2.0867 & 0.2510 & 3.3052 \\
\hline 2014 & 515865.46 & 4.4000 & 64908.63 & 266191.66 & 7.8270 & 84970.55 & 506214.39 & 6.4604 & 2.7237 & 0.2720 & 3.7400 \\
\hline 2015 & 531609.67 & 3,0520 & & & & & & & & & \\
\hline 2016 & 542470.46 & 2,0430 & & & & & & & & & \\
\hline 2017 & 552061.34 & 1,7680 & & & & & & & & & \\
\hline
\end{tabular}

Fuentes: procesamiento propio. Las fuentes y las metodologías de estimación se presentan en el texto.

\section{Referencias}

Aschauer, David Alan (1989). "Is public expenditure productive?", Journal of Monetary Economics, Vol. 23, No. 2, pp. 177-200.

BARro, Robert (1990). "Government spending in a simple model of endogenous growth”, Journal of Political Economy, Vol. 98, No. 5, 103-117.

Cárdenas, Mauricio; Escobar, Andrés \& Gutiérrez, Catalina (1995). “La contribución de la infraestructura a la actividad económica en Colombia”, Ensayos Sobre Política Económica (ESPE), No. 28, pp. 138-187.

CEPAL (1957). Análisis y Proyecciones del Desarrollo Económico (vol. III. Desarrollo Económico de Colombia), México: Naciones Unidas. 
Ortiz, Jiménez y Cruz: El impacto de la infraestructura en el crecimiento económico...

Easterly, William \& Rebelo, Sergio (1993). "Fiscal policy and economic growth: an empirical investigation", NBER Working Paper, No. 4499, National Bureau of Economic Research.

Hall, Robert (1988). "The relation between price and marginal cost in U.S. Industry”, Journal of Political Economy, Vol. 96, No. 5, pp. 921-947.

Hall, Robert \& Jones, Charles (1999). "Why do some countries produce so much output per worker than others?”, Quarterly Journal of Economics, Vol. 114, pp. 83-116.

Harberger, Arnold (1972). "On measuring the social opportunity cost of public funds". En: Project Evaluation-Collected Papers (pp. 42-122). Chicago: The University of Chicago Press.

Hausmann, Ricardo; Hwang, Jason \& Rodrik, Dani (2007). "What you export matters", Journal of Economic Growth, Vol. 12, No. 1, pp. 1-25.

López, Alejandro; Gómez, María Carolina \& Rodríguez, Norberto (1996). "La caída de la tasa de ahorro en Colombia durante los ańos noventa: evidencia a partir de una base de datos para el periodo 1950-1993", Borradores Semanales de Economía, No. 57, Banco de la República, Bogotá.

Ortiz, Carlos Humberto (2016). Diversificación productiva y crecimiento económico: enfoques teóricos y análisis del desarrollo socioeconómico de Colombia. Cali: Programa Editorial de la Universidad del Valle.

Ortiz, Carlos Humberto, Uribe, José Ignacio \& Vivas, Harvy (2013). "Productividad, acumulación y deseconomías públicas en el crecimiento económico colombiano", Cuadernos de Economía, Vol. 32, No. 59, pp. 233263.

Ortiz, Carlos Humberto \& Jiménez, Diana Marcela (2016). "A Smithian analysis of the Colombian economic growth", Ensayos Sobre Politica Económica, Vol. 34, No. 79, pp. 66-77.

Ortiz, Carlos Humberto \& Jiménez, Diana Marcela (2017). “Un análisis smithiano del crecimiento económico colombiano: avances metodológicos", Lecturas de Economia, No. 87, pp. 35-66. 
Pachón, Álvaro \& Ramírez, María Teresa (2006). La infraestructura de transporte en Colombia durante el siglo XX. Bogotá: Fondo de Cultura Económica - Banco de la República.

Palei, Tatyana (2015). "Assessing the impact of infrastructure on economic growth and global competitiveness", Procedia Economics and Finance, Vol. 23, pp. 168-175.

Perdomo, Álvaro Andrés (2002). "Inversión pública sectorial y crecimiento económico: una aproximación desde la metodología VAR", Archivos de Economía, No. 002921. Departamento Nacional de Planeación, Colombia.

Posada, Carlos Esteban \& Gómez, William (2002). “Crecimiento económico y gasto público: un modelo para el caso colombiano", Ensayos sobre Politica Económica, Vol. 20, No. 41-42.

Poveda Ramos, Gabriel (2005). Historia económica de Colombia en el siglo XX. Medellín: Universidad Pontificia Bolivariana, Medellín.

Rebelo, Sergio (1991). "Long-run policy analysis and long-run growth", Journal of Political Economy, Vol. 99, No. 3, pp. 500-521.

Roberts, Mark J. (1989). "Structure of production in Colombian manufacturing industries 1977-1985”. Reporte del proyecto de investigación Industrial competition, productive efficiency, and their relation to trade regimes. Banco Mundial.

RoDrik, Dani (2007). "Industrial development: some stylized facts and policy directions". En: Industrial Development for the 21st Century: Sustainable Development Perspectives (pp. 7-28). New York: United Nations.

SÁnCheZ, Fabio; Rodríguez, Jorge Iván \& NúÑEZ, Jairo (1996). "Evolución y determinantes en Colombia: un análisis global y sectorial, 1950-1994", En: Chica, R. (Coordinador), El crecimiento de la productividad en Colombia: resultados del estudio nacional sobre determinantes del crecimiento de la productividad. Bogotá: DNP, COLCIENCIAS, FONADE. 
Ortiz, Jiménez y Cruz: El impacto de la infraestructura en el crecimiento económico...

Smith, Adam (1958). Investigación sobre la naturaleza y causas de la riqueza de las naciones. México: Fondo de Cultura Económica (trabajo original publicado en 1776).

Solow, Robert Merlon (1957). "Technical change and the aggregate production function", The Review of Economics and Statistics, Vol. 39, No. 3, pp. 312-320. 